\title{
Sensitivity of Greenland ice sheet simulation to the numerical procedure employed for ice-sheet dynamics
}

\author{
Fuyuki SAITO, ${ }^{1,2}$ Ayako ABE-OUCHI ${ }^{1}$ \\ ${ }^{1}$ Center for Climate System Research, The University of Tokyo, Komaba 4-6-1, Meguro-ku, Tokyo 153-8904, Japan \\ E-mail: saitofuyuki@jamstec.go.jp \\ ${ }^{2}$ Japan Agency fopr Marine-Earth Science and Technology, Frontier Research Center for Global Change, \\ 3173-25 Showamachi, Kanazawa, Yokohama 236-0001, Japan
}

\begin{abstract}
The response of the Greenland ice sheet to global warming is simulated by two different numerical approaches, in order to evaluate the sensitivity of the analysis to the numerical structure employed. It is found that the thickness near the margin differs appreciably in these two simulations under identical conditions of modest warming, primarily due to a significant increase in the warming effect by an elevation-ablation feedback mechanism in one of the simulations. The change in ice-sheet volume differs by as much as a factor of two under strong climate-change forcing, demonstrating the need for care in interpreting the results of such climate-change analysis.
\end{abstract}

\section{INTRODUCTION AND BACKGROUND}

To discuss the response of the Greenland ice sheet to global warming and its contribution to sea-level rise using a numeri-cal ice-sheet model, it is necessary to investigate several uncertainties related to the model itself. The uncertainties arise from imperfect modeling of physical processes such as basal sliding, ice stiffness, ablation and calving (Ritz and others, 1997). Moreover, numerical procedures such as model resolution affect the uncertainties (Ritz and others, 1997). Hindmarsh and Payne (1996) and Huybrechts and others (1996) have shown using an ideal isothermal ice-sheet model that the numerical procedure itself introduces a certain margin of error into simulation. This paper therefore discusses how simulation of the response of the ice sheet to warming is influenced by the numerical structure employed.

Ice-sheet models can be divided into two main groups based on the numerical methods used for mass-balance calculations (Hindmarsh and Payne, 1996; Huybrechts and others, 1996). Typically, the mass-balance equation in the flux form,

$$
\frac{\partial H}{\partial t}=-\nabla \cdot \vec{q}+m_{\mathrm{s}}
$$

is rewritten in diffusion form as follows:

$$
\frac{\partial H}{\partial t}=-\nabla \cdot(D \nabla H)-\nabla \cdot(D \nabla b)+m_{\mathrm{s}}
$$

where $H$ is the ice thickness, $b$ is the bedrock elevation, $\vec{q}$ is the ice-flux vector and $m_{\mathrm{s}}$ is the surface mass-balance term (accumulation minus ablation). The term $D$ is a non-linear diffusion term that is calculated under the shallow-ice approximation as follows:

$$
\begin{aligned}
D=- & 2(\rho g)^{n}\left[\left(\frac{\partial h}{\partial x}\right)^{2}+\left(\frac{\partial h}{\partial y}\right)^{2}\right]^{\frac{n-1}{2}} \\
& \int_{b}^{h} \mathrm{~d} z \int_{b}^{z} \mathrm{~d} z^{\prime} m A(T)\left(h-z^{\prime}\right)^{n},
\end{aligned}
$$

where $\rho$ is the ice density, $g$ is gravity acceleration, $h$ is surface elevation, $A$ is the temperature-dependent rate factor, and $m$ is the enhancement factor. The ice flux $\vec{q}$ is evaluated in terms of $D$ as

$$
\vec{q}=D \nabla(H+b)
$$

In the horizontal discretization of Equation (1), the $q$ term is typically evaluated on the mid $x\left(q_{i+1 / 2, j}^{x}\right)$ and $y\left(q_{i, j+1 / 2}^{y}\right)$ points. Thus, using the second-order central difference and the staggered grid system, the evolution of thickness at gridpoint $(i, j)$ requires four terms: $D_{i-1 / 2, j}, D_{i+1 / 2, j}, D_{i, j-1 / 2}$ and $D_{i, j+1 / 2}$, as given by Equation (2). In one of the methods presented in Hindmarsh and Payne (1996), $D_{i+1 / 2, j}$ is evaluated directly using a staggered grid as follows:

$$
D_{i+\frac{1}{2}, j}=F_{i+\frac{1}{2^{\prime}}, j} \times\left[\left.\left.\nabla h\right|_{i+\frac{1}{2^{\prime}}, j} \cdot \nabla h\right|_{i+\frac{1}{2^{\prime}}, j}\right]^{\frac{n-1}{2}},
$$

where

$$
\begin{gathered}
F_{i, j}=-2(\rho g)^{n} \int_{b_{i, j}}^{h_{i, j}} \mathrm{~d} z \int_{b_{i, j}}^{z} \mathrm{~d} z^{\prime} m A(T)\left(h_{i, j}-z^{\prime}\right)^{n}, \\
\nabla h \cdot \nabla h=\left(\frac{\partial h}{\partial x}\right)^{2}+\left(\frac{\partial h}{\partial y}\right)^{2} .
\end{gathered}
$$

In another method, $D_{i+1 / 2, j}$ is evaluated by the average of two adjacent gridpoints, as given by

$$
\begin{aligned}
D_{i+\frac{1}{2}, j}= & \frac{1}{2}\left(D_{i, j}+D_{i+1, j}\right) \\
= & \frac{1}{2}\left\{F_{i, j} \times\left[\left.\left.\nabla h\right|_{i, j} \cdot \nabla h\right|_{i, j}\right]^{\frac{n-1}{2}}\right. \\
& \left.+F_{i+1, j} \times\left[\left.\left.\nabla h\right|_{i+1, j} \cdot \nabla h\right|_{i+1, j}\right]^{\frac{n-1}{2}}\right\} .
\end{aligned}
$$

A similar treatment can also be used for $D_{i, j+1 / 2}$. Adopting the second-order central difference for computation of the surface gradient, the first method uses a total of 9 adjacent points to compute one gridpoint, whereas the second method uses 13 points. Thus, the two methods are referred to here as the 9-point scheme and the 13-point scheme, respectively. The two methods are illustrated in Figure 1. The 9-point scheme, deriving from the method of Mahaffy (1976), is mass-conserving but has poor stability properties. In contrast, the 13-point scheme, derived originally from Huybrechts (1992), is stable for much larger time-steps (Huybrechts and 
(a) 9-point scheme

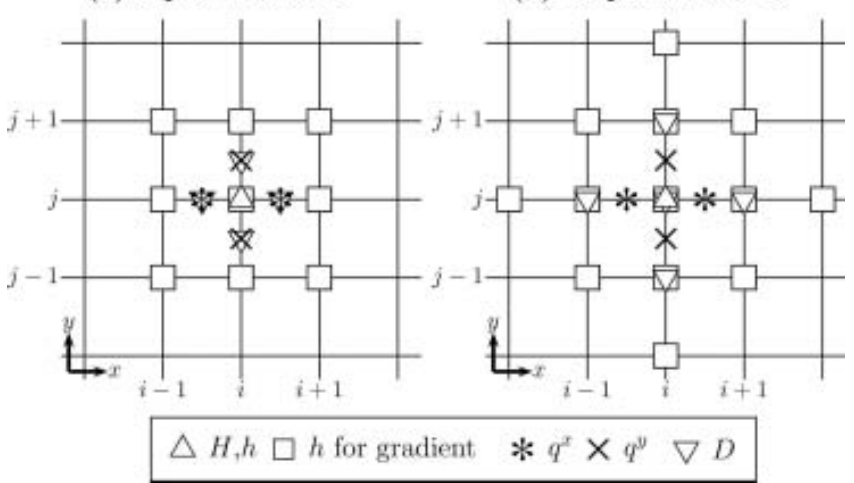

Fig. 1. Models for evaluation of $D$. Indices $i$ and $j$ are grid number of the model domain in the $x$ and $y$ axes, respectively. Symbols are gridpoints to evaluate each term. The term $H$ is ice thickness, $h$ is surface elevation, $q^{x}$ and $q^{y}$ are horizontal flux, and $D$ is the nonlinear diffusion term (see text).

others, 1996), but opinions differ on its mass-conservation properties (Hindmarsh and Payne (1996) maintain that it is mass-conserving, while Huybrechts and others (1996) state that it is not). The present study compares the two schemes without discussing the relative performance. Hindmarsh and Payne (1996) reported that for an isothermal ice-sheet model the 9-point scheme overestimates the steady-state thickness while the 13-point scheme gives an underestimate compared to the analytical (exact) solution.
It is important to note that in both cases the error increases towards the margin. As ablation is generally active near the margin due to the higher temperatures that occur at lower elevation, the simulated response is directly affected by the error near the margin. This becomes a particularly important consideration in global warming experiments with elevation-ablation feedback, i.e. the lower the elevation the more melting occurs. Sensitivity experiments have been conducted by a number of authors for the Greenland ice sheet under spatially uniform background warming (Letréguilly and others, 1991; Abe-Ouchi, 1993; Fabre and others, 1995; Greve, 2000), and it has generally been shown that the ice sheet exhibits a non-linear response to temperature shifts, with warming of $5-6 \mathrm{~K}$ required to reach complete melting of the Greenland ice sheet. However, these previous analyses adopted only one of the numerical schemes without comparison with alternative schemes. For example, Abe-Ouchi (1993) employed an analogous two-dimensional 9-point scheme, while Letréguilly and others (1991) and Greve (2000) applied a 13-point scheme.

Huybrechts and others (1996) discussed the differences between simulations using two different schemes for ten isothermal models under ideal boundary conditions and geometry. However, ablation is a function of position only, and not affected by changes in elevation. Moreover, the timestep and vertical resolution can be chosen arbitrarily by individual modelers. Thus, there has been no direct comparison between two models of three-dimensional and thermomechanical coupling for a given scenario. In the present study, two different schemes are employed for simulation of
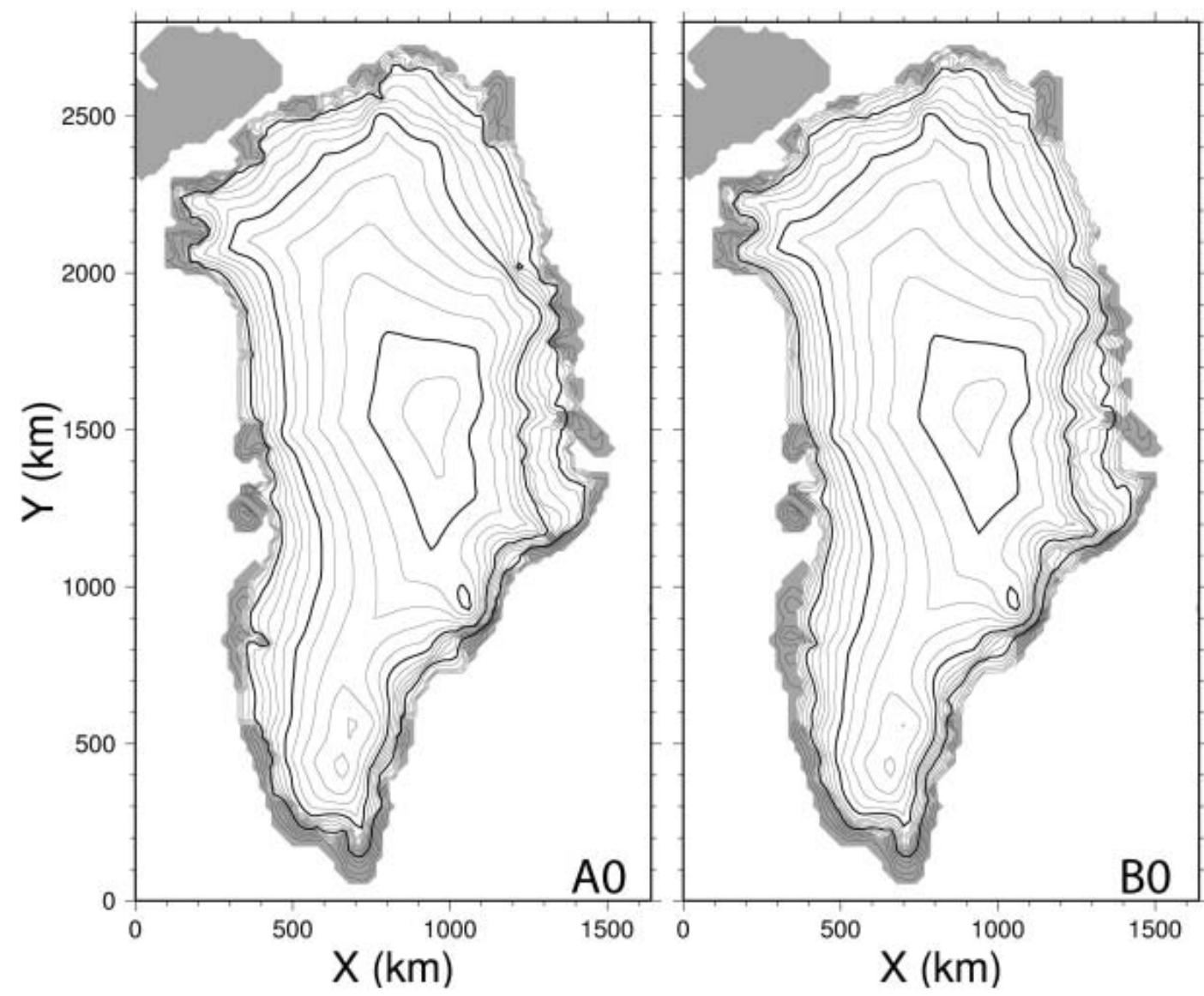

Fig. 2. Final state reached in the control experiments A0 (left) and B0 (right). Surface topography is shown in contour intervals of $200 \mathrm{~m}$ (thin) and $1000 \mathrm{~m}$ (thick), with ice-free land indicated by shading. 
the response of the Greenland ice sheet to global warming in order to evaluate the uncertainties in the simulated response due solely to the numerical scheme applied.

\section{MODEL DESCRIPTION}

The ice-sheet model used in the present work is a standard three-dimensional shallow-ice approximation model similar to that presented in Saito (2002) and Saito and Abe-Ouchi (2004). The model computes the evolution of ice thickness, bedrock and ice temperature under the prescribed scenario of climate forcing. The semi-implicit scheme (Hindmarsh and Payne, 1996) is applied for solving the mass-balance equation (2).

The dependence of ice rheology on the computed ice temperature is given by Huybrechts (1992):

$$
A\left(T^{\prime}\right)=a \exp \left(\frac{-Q}{R T^{\prime}}\right)
$$

where $T^{\prime}$ is the absolute temperature corrected for the dependence of the melting point on pressure (Paterson, 1994), and a and $Q$ are given by

$$
\left.\begin{array}{l}
\begin{array}{l}
a=3.61 \times 10^{-13} \mathrm{~Pa}^{-3} \mathrm{~s}^{-1} \\
Q
\end{array}=1.73 \times 10^{3} \mathrm{~J} \mathrm{~mol}^{-1}
\end{array}\right\} \quad \text { if } T^{\prime}<263.15 \mathrm{~K},
$$

The bedrock and surface topography compiled by Letréguilly and others (1991) is employed on a $20 \mathrm{~km}$ grid spacing using a polar stereographic projection. This topography dataset is distributed as part of the European Ice Sheet Modelling Initiative (EISMINT) for comparison of the Greenland ice sheet (coordinated by C. Ritz; see Huybrechts, 1998). The horizontal model domain spans $1640 \times 2800 \mathrm{~km}^{2}$ with a grid resolution of $20 \mathrm{~km}$ in both horizontal coordinate directions ( $83 \times 141$ gridpoints). The vertical grid is divided equally into 30 levels. Different time-steps are employed for solving the dynamic evolution ( $\Delta t=4.0$ years) and the thermodynamic evolution ( $\Delta t=20.0$ years). The dynamics of the ice shelf and the grounding line are not included in the present model. The lateral boundary is decided by a floating condition by which any part of the ice sheet that floats due to its own buoyancy is immediately cut off. The geothermal heat flux for the boundary condition of thermodynamics is set at a constant value of $42 \mathrm{~mW} \mathrm{~m}^{-2}$ throughout the experiments (Lee, 1970). The ice enhancement factor is set at 3, and basal sliding is ignored. Changes in the elevation of the glacier bed under the load of the ice are calculated using an equation expressing the local isostatic rebound with a time constant of 3000 years (Turcotte and Schubert, 1982). Monthly observed data compiled by Calanca and others (2000) are used for the annual surface temperature, which is perturbed according to both the warming scenario and local surface elevation changes in terms of the prescribed lapse rate of $6.5 \mathrm{~K} \mathrm{~km}^{-1}$. Computation took about $20 \mathrm{~min}$ per $50 \mathrm{kyr}$ on a Hitachi SR8000 supercomputer.

The second part of the present model calculates the surface mass balance in two parts, accumulation and ablation, which are parameterized separately. Surface accumulation follows the present observation compiled by Calanca and others (2000). It is assumed that there is no

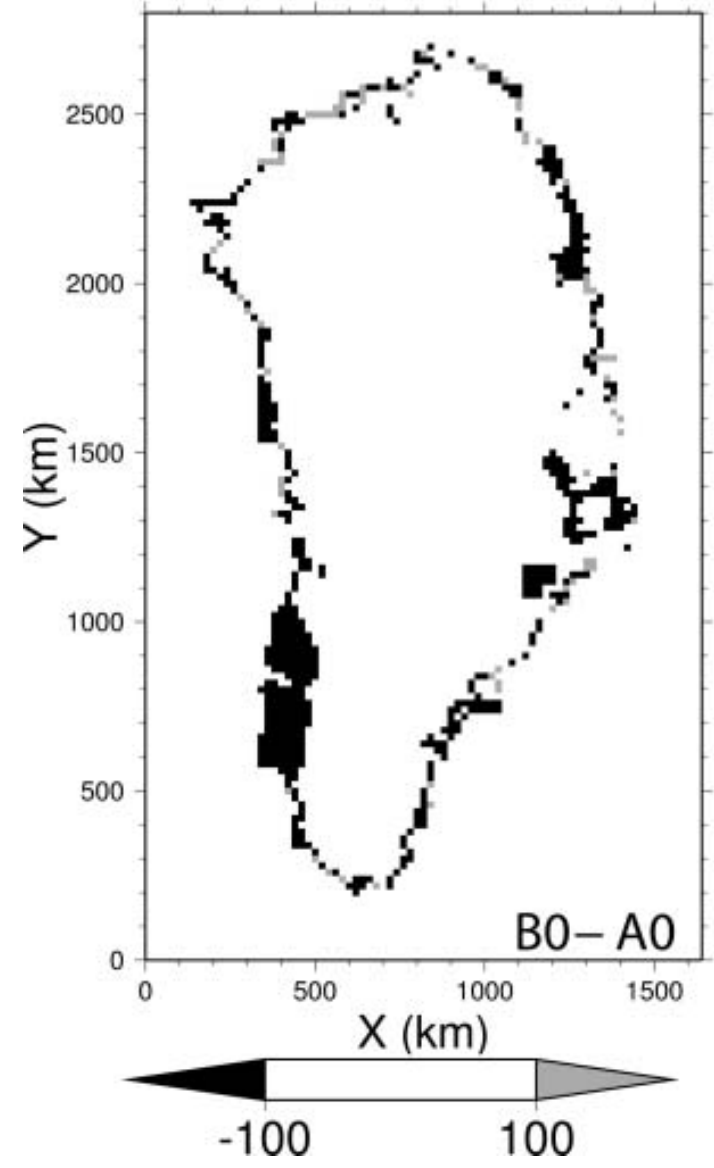

Fig. 3. Difference between the surface topography of experiments $\mathrm{BO}$ and $\mathrm{AO}$ in the final state.

change in the accumulation distribution. Although ablation depends on the details of the energy balance at the ice-sheet surface, it is not possible to apply full energy-balance calculations over the entire model domain at every timestep. Therefore, ablation is parameterized by a simple function relating surface temperature to the ablation (the physical basis for this parameterization has been discussed by Ohmura (2001)). The function in this paper assumes the following relation between summer temperature $T_{\mathrm{JJA}}$ at the surface and ablation $a_{\mathrm{bl}}\left(\right.$ in $\mathrm{mm}$ w.e. $\mathrm{a}^{-1}$ ):

$$
a_{b l}=\max \left[0,\left(500 T_{J J A}+1000\right)\right],
$$

based on extensive observations in Greenland (Ohmura and others, 1996). Equation (14) indicates that $a_{b l}=0$ when $T_{\mathrm{JA}} \leq-2^{\circ} \mathrm{C}$, and captures the observed features well. Ablation calculation requires the mean summer temperature or time series of surface temperature. In the present work, monthly observed data compiled by Calanca and others (2000) were used for the reference temperature, which was then perturbed by warming and local elevation change. Most previous studies have used the positive-degree-day method (Letréguilly and others, 1991), and Saito (2002) has discussed the difference between the two parameterizations. Although the results of the simulations using the two parameterizations differ, the main conclusion of this paper is not significantly affected.

\section{EXPERIMENT AND RESULTS}

The initial condition for the sensitivity studies was defined as the observed surface and bedrock topographies and an 

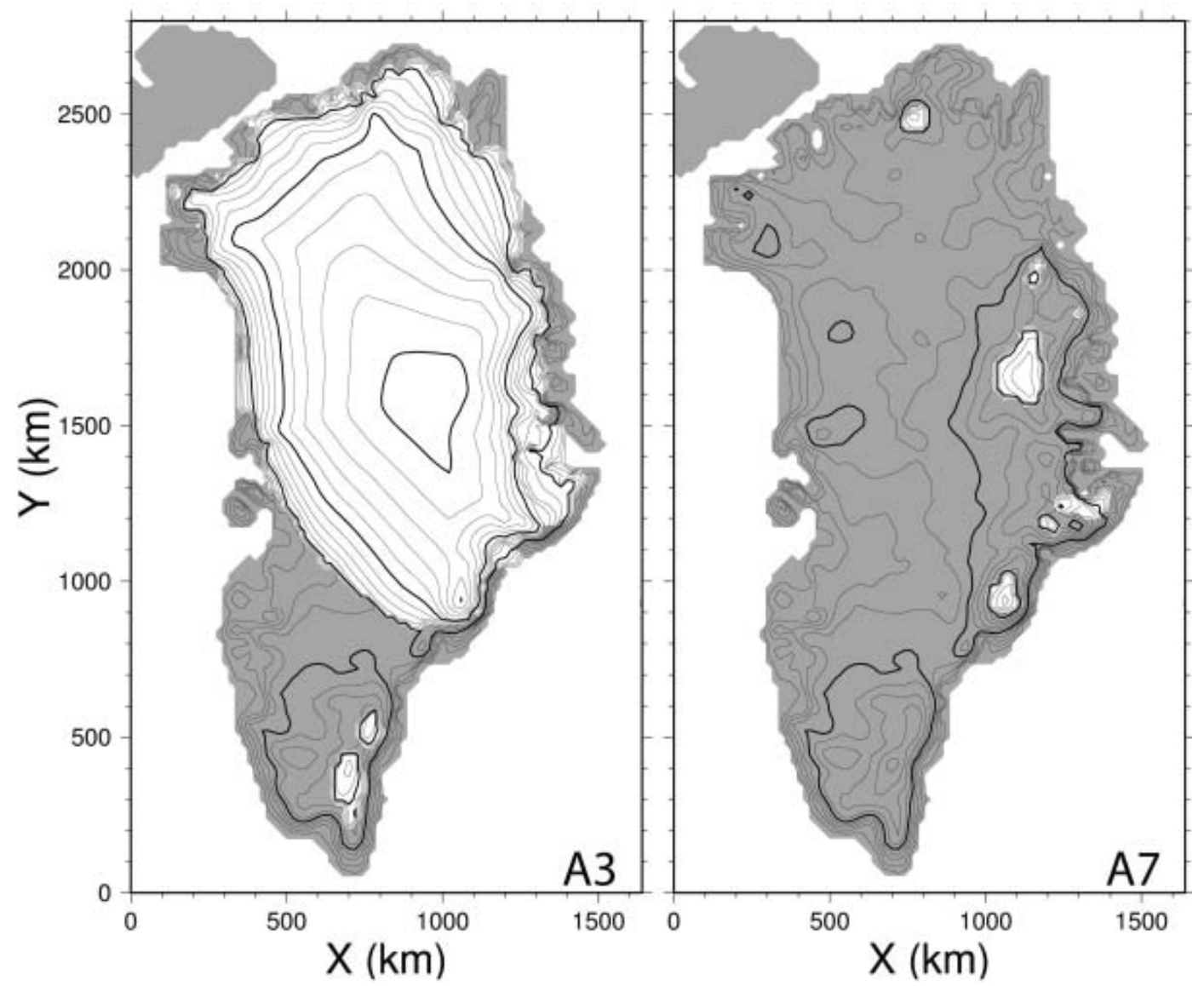

Fig. 4. Final results of A3 (left) and A7 (right), showing surface topography in contour intervals of $200 \mathrm{~m}$ (thin) and $1000 \mathrm{~m}$ (thick). Ice-free land is shaded.

isothermal ice sheet. Using this initial condition, a simulation with fixed topography and free thermodynamics was conducted first under the present climate-forcing condition for $100 \mathrm{kyr}$. Then, using the final state of that simulation as the new initial condition, a further simulation with free topography was run for $100 \mathrm{kyr}$. The final state of this second simulation was then used as the initial condition for all

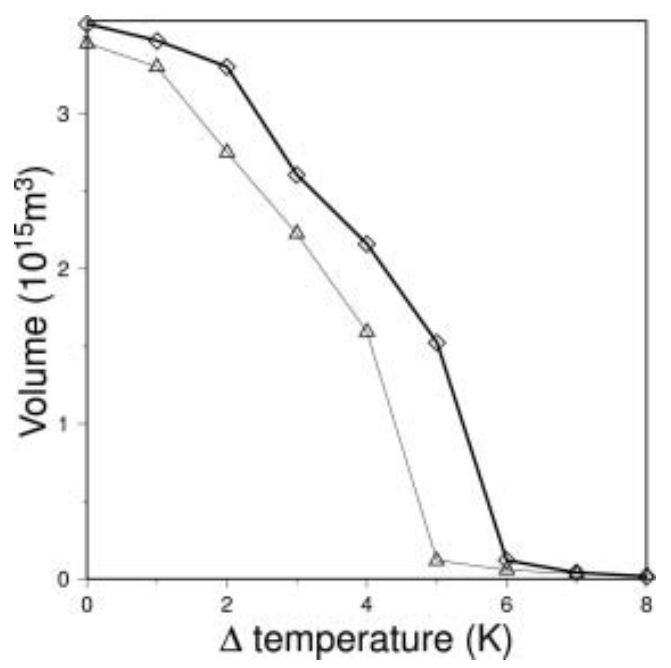

Fig. 5. Final volume change in response to warming scenarios. Thick and thin lines are the results of experiment A and B series, respectively. sensitivity studies. Initial conditions were prepared using this model for each of the two numerical schemes.

Uniform stepwise warming, where a spatially constant perturbation is applied suddenly to the initial condition, was adopted for all sensitivity studies. Experiments A and B were performed using the 9-point and 13-point scheme, respectively. A total of nine sensitivity experiments were conducted for each numerical scheme, with warming of +0 , $+1, \ldots,+8 \mathrm{~K}$ (referred to as experiments $\mathrm{A} 0, \mathrm{~A} 1, \ldots, \mathrm{A} 8$ etc.). All sensitivity studies were run for $50 \mathrm{kyr}$, sufficient to reach a steady state. Figure 2 shows the results for the control experiments $\mathrm{A} 0$ and $\mathrm{B} 0$. In both cases, the surface elevation (highest position) is well simulated compared to the observations. However, while the model simulates the observed features in the interior region quite well, the elevation (or thickness) near the margin is overestimated by $400 \mathrm{~m}$ or more. The ice-sheet coverage is also overestimated by the simulation, which produces an overextension of the ice sheet at the northeast and southwest margins. Thus, the icesheet volume is also overestimated. The steady-state volumes indicated by $\mathrm{A} 0$ and $\mathrm{B} 0$ are $3.58 \times 10^{6}$ and $3.45 \times 10^{6} \mathrm{~km}^{3}$, respectively, $38 \%$ and $33 \%$ higher than the present measured value of $2.6 \times 10^{6} \mathrm{~km}^{3}$ (obtained from Allison and others, 2006).

This overestimation is a common feature of many numerical simulations of the Greenland ice sheet (Ritz and others, 1997). Ritz and others (1997) concluded that a high ablation rate is necessary to obtain good agreement between the simulations and observations. However, other parameters may also have an effect. For example, ignoring basal sliding 

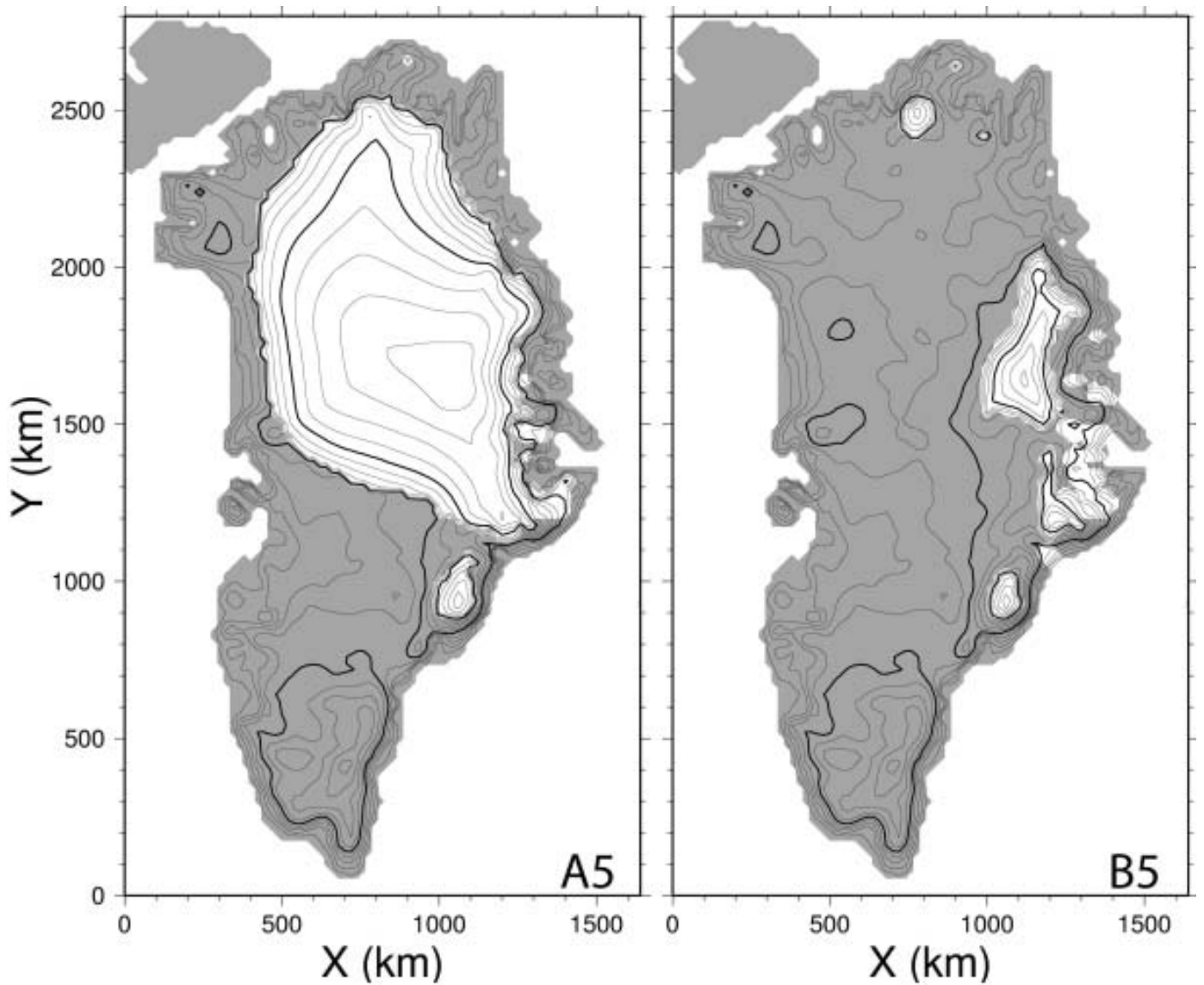

Fig. 6. Final result of $5 \mathrm{~K}$ warming for experiments $\mathrm{A} 5$ (left) and B5 (right), showing surface topography in contour intervals of $200 \mathrm{~m}$ (thin) and $1000 \mathrm{~m}$ (thick). Ice-free land is shaded.

tends to produce thicker ice-sheet margins, and the presence of ice streams lowers the ice stiffness and increases the ice flux due to higher-order stress effects (not introduced under the shallow-ice approximation), basal sliding and larger strain heating. However, these effects cannot be represented in the present model. Unknown parameters such as geothermal heat flux or enhancement factors are also difficult to express as a single parameter for all of Greenland. Furthermore, the present ice sheet may not necessarily be in a steady state, and may in fact be in transition responding to past climate variability. All or some of these effects may cause an overall bias in the result. For the purposes of the present study, however, these overestimations are not important. Figure 3 shows the difference between the surface elevations reached in the final state $(50 \mathrm{kyr})$ in the two control experiments $\mathrm{AO}$ and $\mathrm{BO}$ ( $\mathrm{B} 0$ minus $\mathrm{AO}$ is shown). As explained by Hindmarsh and Payne (1996) using an isothermal ice-sheet model, the 13-point scheme gives a solution in which the surface elevation near the margin is lower, in this case by $>200 \mathrm{~m}$. On the other hand, the difference in interior regions is very small.

Figure 4 shows the final surface topography obtained by experiments A3 and A7. The response patterns of the Greenland ice sheet to uniform warming in both cases are generally similar, revealing a fragile region in the southwest between the two summits. The ice sheet separates into two parts under relatively small warming of $3 \mathrm{~K}$, while under higher forcing of around $+5 \mathrm{~K}$ the southern part disappears entirely, leaving only the northern area. With further forcing, the northern region also begins to disappear, finally leaving only a small area on the higher mountains in the east. Although the mass-balance parameterizations differ, the results of this paper are comparable to those obtained in previous studies (Letréguilly and others, 1991).

Figure 5 shows the final volume $(50 \mathrm{kyr})$ obtained by these experiments. Although the volume change is modest for small temperature shifts, the gradient of volume decrease becomes steeper with increasing temperature shift. The gradient becomes steepest with $5 \mathrm{~K}$ warming, and almost all the ice melts with higher warming. However, while warming of $5 \mathrm{~K}$ results in a loss of approximately half the volume of the Greenland ice sheet in A5, the same warming causes most of the ice sheet to disappear in B5 (Fig. 6). The uncertainty of the temperature perturbation that causes most of the ice sheet to disappear is about $1 \mathrm{~K}$.

The major reason for the difference in the response is the difference between the two control experiments. As ablation is most where the elevation is lowest (e.g. the margin), the sensitivity to warming increases as the surface elevation decreases. As a result of elevation-ablation feedback, the higher margin in A0 compared to B0 offsets the simulated response by about $1 \mathrm{~K}$.

The uncertainty evaluated in this paper is limited to models with horizontal resolution of $20 \mathrm{~km}$. As explained by Hindmarsh and Payne (1996) using an isothermal ice-sheet model, the solutions obtained by these two methods approach the exact solution from opposite sides at the high horizontal resolution employed here.

The volume-temperature diagram (Fig. 5) for the present Greenland ice sheet will shift leftwards (in the direction of 
lower temperature): As the elevation of the margin is overestimated in all of the cases examined in this study, the response will also be underestimated in all of the present cases. Moreover, the change in surface albedo due to the expansion of ice-free land may increase the sensitivity of the response. However, even if these processes are included in the model, a degree of uncertainty remains in either of these numerical analyses of the sensitivity of the ice-sheet volume to global warming.

\section{CONCLUSION}

The response of the Greenland ice sheet to global warming was evaluated using a three-dimensional ice-sheet model and two different numerical schemes in order to determine the sensitivity of the simulation to the numerical procedure employed. It was shown that the response of the Greenland ice sheet is influenced strongly by this difference in the numerical structure adopted for thickness evolution. In estimation of the contribution of ice-sheet melting to future sea-level rise, the steady state differs by as much as a factor of two under the same climate-change scenario $(+5 \mathrm{~K}$ in this study). Thus, the uncertainty in the temperature perturbation required for a certain volume change is about $1 \mathrm{~K}$ in the $2-5 \mathrm{~K}$ warming range. As the realistic range of future and past warming is within this range, careful interpretation of simulated volume change is required, and the adequacy of the numerical scheme employed should be considered in more detail. These topics will be addressed in future research.

\section{ACKNOWLEDGEMENTS}

The authors thank R. Warner and an anonymous referee for valuable comments on the manuscript. This work was supported by MEXT KAKENHI 17740301.

\section{REFERENCES}

Abe-Ouchi, A. 1993. Ice-sheet response to climate changes: a modelling approach. Zürcher Geogr. Schr. 54.

Allison, I., R.G. Barry and B.E. Goodison, eds. 2006. Climate and Cryosphere (CliC) Project. Science and Co-ordination Plan, Version 1, WCRP-114, WMO/TD No. 1053. Geneva, World Climate Research Programme.
Calanca, P., H. Gilgen, S. Ekholm and A. Ohmura. 2000. Gridded temperature and accumulation distributions for Greenland for use in cryospheric models. Ann. Glaciol., 31, 118-120.

Fabre, A., A. Letréguilly, C. Ritz and A. Mangeney. 1995. Greenland under changing climates: sensitivity experiments with a new three-dimensional ice-sheet model. Ann. Glaciol., 21, 1-7.

Greve, R. 2000. On the response of the Greenland ice sheet to greenhouse climate change. Climatic Change, 46(3), 289-303.

Hindmarsh, R.C.A. and A.J. Payne. 1996. Time-step limits for stable solutions of the ice-sheet equation. Ann. Glaciol., 23, 74-85.

Huybrechts, P. 1992. The Antarctic ice sheet and environmental change: a three-dimensional modelling study. Ber. Polarforsch. 99.

Huybrechts, P. 1998. Report of the third EISMINT Workshop on model intercomparison. Strasbourg, European Science Foundation.

Huybrechts, P., T. Payne and the EISMINT Intercomparison Group. 1996. The EISMINT benchmarks for testing ice-sheet models. Ann. Glaciol., 23, 1-12.

Lee, W.H.K. 1970. On the global variations of terrestrial heat flow. Phys. Earth Planet. Inter., 2(5), 332-341.

Letréguilly, A., P. Huybrechts and N. Reeh. 1991. Steady-state characteristics of the Greenland ice sheet under different climates. J. Glaciol., 37(125), 149-157.

Mahaffy, M.W. 1976. A three-dimensional numerical model of ice sheets: tests on the Barnes Ice Cap, Northwest Territories. J. Geophys. Res., 81(6), 1059-1066.

Ohmura, A. 2001. Physical basis for the temperature-based meltindex method. J. Appl. Meteorol., 40(4), 753-761.

Ohmura, A., M. Wild and L. Bengtsson. 1996. A possible change in mass balance of Greenland and Antarctic ice sheets in the coming century. J. Climate, 9(9), 2124-2135.

Paterson, W.S.B. 1994. The physics of glaciers. Third edition. Oxford, etc., Elsevier.

Ritz, C., A. Fabre and A. Letréguilly. 1997. Sensitivity of a Greenland ice sheet model to ice flow and ablation parameters: consequences for the evolution through the last glacial cycle. Climate Dyn., 13(1), 11-24.

Saito, F. 2002. Development of a three dimensional ice sheet model for numerical studies of Antarctic and Greenland ice sheet. Tokyo, University of Tokyo. Center for Climate System Research. (CCSR Report 15.)

Saito, F. and A. Abe-Ouchi. 2004. Thermal structure of Dome Fuji and East Queen Maud Land, Antarctica, simulated by a threedimensional ice sheet model. Ann. Glaciol., 39, 433-438.

Turcotte, D.L. and G. Schubert. 1982. Geodynamics: applications of continuum physics to geological problems, New York, etc., John Wiley and Sons. 